\title{
Effect of Nonparallel End Face on Energy Dissipation Analyses of Rocklike Materials Based on SHPB Tests
}

\author{
Pu Yuan $\mathbb{D}^{1,2,3}$ Ning-Ning Wei ${ }^{1}{ }^{1}$ and Qin-Yong Ma $\mathbb{D}^{1,2,3}$ \\ ${ }^{1}$ School of Civil Engineering and Architecture, Anhui University of Science and Technology, Huainan 232001, China \\ ${ }^{2}$ State Key Laboratory of Mining Response and Disaster Prevention and Control in Deep Coal Mines, \\ Anhui University of Science and Technology, Huainan 232001, China \\ ${ }^{3}$ Engineering Research Center of Underground Mine Construction, Ministry of Education, \\ Anhui University of Science and Technology, Huainan 232001, China
}

Correspondence should be addressed to Pu Yuan; puy2012@126.com

Received 9 May 2019; Accepted 2 July 2019; Published 18 July 2019

Academic Editor: Itzhak Green

Copyright $(2019 \mathrm{Pu}$ Yuan et al. This is an open access article distributed under the Creative Commons Attribution License, which permits unrestricted use, distribution, and reproduction in any medium, provided the original work is properly cited.

To evaluate the effect of nonparallel end face of rocklike specimens in SHPB tests, the characteristics of energy dissipation are analyzed based on numerical simulations for end-face nonparallelism from $0 \%$ to $0.40 \%$ and Young's modulus from $14 \mathrm{GPa}$ to $42 \mathrm{GPa}$. With the increment of end-face nonparallelism, both energy consumption density and dissipated energy density show a slight increase trend, while releasable elastic strain energy density presents a slight decrease trend. Existence of elastic unloading in the damaged rocklike specimen leads to a reduction of energy consumption density and a constant dissipated energy density during total strain shrinkage. At peak dynamic stress, dissipated energy density presents a linear upward trend with the increment of end-face nonparallelism and Young's modulus, while releasable elastic strain energy density shows an inverse trend. A binary linear regression equation is deduced to estimate the energy dissipation ratio. Mechanical damage evolution of the rocklike specimen is divided into two regions in line with the two regions in dynamic stress-strain curves, and the transition between the slow-growth region and rapid-growth region is shifted to the right with the increment of end-face nonparallelism. Due to the presence of nonparallel end face, fluctuation presents in energy density evolution and mechanical damage evolution. The fluctuation is enhanced with the increment of end-face nonparallelism and weakened with the increase of Young's modulus. Based on energy density evolution and mechanical damage evolution analyses, the maximum end-face nonparallelism should be controlled within $0.20 \%$, twice the value in ISRM suggested methods, which reduces the cost and time for processing rocklike specimens.

\section{Introduction}

The split Hopkinson pressure bar (SHPB), also known as Kolsky bar, is an extensive, convenient, and reliable technique to characterize the behaviors of solid materials at a high strain rate, mainly in the range of $10^{2}-10^{4} \mathrm{~s}^{-1}$ [1]. It is widely used to quantify the dynamic properties of metallic materials $[2,3]$. Then, it is generalized to brittle material, such as concrete [4], ceramics [5], frozen soil [6], and rocks $[7,8]$. To overcome the major limitations of SHPB apparatus for rocklike materials, pulse-shaping technique or changing the shape of the striker is employed to generate a ramped incident stress wave [9], and some fundamental issues in the
SHPB test are also analyzed for rocklike materials [10]. Test methods based on the SHPB apparatus are suggested by ISRM to determine the dynamic properties of the rock material [11].

With the development of SHPB technique, the accuracy and precision of dynamic mechanical characteristics are the key issues for SHPB tests. Based on numerical simulations, six types of incident bar misalignment in SHPB apparatus are investigated, and the distorted signal generated by bar misalignment is mainly induced by the presence of flexural modes of vibration and affects the SHPB test results adversely [12]. To evaluate the effects of imperfect condition on incident stress waves, both experiments and numerical 
simulations are carried out, and the inclination and indentation of impact end-surface show a great impact on incident stress waves [13]. For a physical SHPB apparatus, the errors induced by imperfect impact interface and bar misalignment can be minimized and eliminated by aligning and manufacturing the bars precisely. In SHPB tests, the pending tested specimen is sandwiched between the incident bar and transmitted bar. Considering the processing accuracy of the specimen, the specimen geometry imperfections on SHPB tests for ductile materials are analyzed from the reflected wave, transmitted wave, and dynamic stress-strain curve, and the error induced by specimen geometry imperfections is small for ductile materials and can be neglected when imperfection angles no larger than $0.3^{\circ}[14]$. While for rocklike materials, the specimen geometry imperfections in SHPB test is more adverse than ductile martials. When end-face nonparallelism is within $0.40 \%$, the nonparallel end face shows a small and negligible influence on dynamic stress, while it shows a great impact on dynamic strain and strain rate [15]. According to ISRM suggested test methods [11], cylinder rock specimens with diameter of $50 \mathrm{~mm}$ and length-to-diameter ratio of 0.5 are widely used in SHPB tests. Each rock specimen is processed through drilling, cutting, and grinding processes [16]. For short cylinder rock specimen, the processing accuracy and precision are very difficult to control, especially the perpendicularity of two ends to the axis. Besides, high processing accuracy and precision of rock specimens are always related with expensive manufacturing technologies and long processing time. By analyzing the end-face nonparallel effect in the SHPB test, the allowable processing deviation is investigated to reduce the cost and save the processing time without affecting the reliability of SHPB tests.

The deformation and failure of rock can be considered as an irreversible process of energy dissipation [17]. Therefore, energy dissipation of the rock under dynamic loads can be studied based on SHPB tests [18] and is becoming a major issue in rock mechanics and rock engineering $[19,20]$.

Considering the processing deviation of the rocklike specimen, numerical simulations of SHPB tests are conducted for nonparallel end-face rocklike specimens with various Young's moduli by LS-DYNA. During numerical simulation, end-face nonparallelism ranges from $0.0 \%$ to $0.40 \%$, and Young's modulus ranges from $14 \mathrm{GPa}$ to $42 \mathrm{GPa}$. Then, energy density dissipation is analyzed to reveal the effect of end-face nonparallellism. As mechanical damage evolution is closely related with energy dissipation, the influence of nonparallel end face on mechanical damage evolution is also studied.

\section{Setup of 3D Numerical Model for SHPB Test and Verification}

2.1. Setup of $3 D$ Numerical Model. Based on the physical $\Phi 50 \mathrm{~mm}$ SHPB apparatus, a series of 3D finite element models without a striker are set up to conduct SHPB tests for rocklike materials. Basically, a typical SHPB apparatus consists of a striker, an incident bar, and a transmitted bar. As shown in Figure 1, an incident bar, a rocklike specimen, and a transmitted bar are considered and built in a 3D finite element model, and the nonparallel end face of the rocklike specimen is contacted with the transmitted bar. The length and diameter for both incident and transmitted bars are $2000 \mathrm{~mm}$ and $50 \mathrm{~mm}$, respectively. In numerical simulation of SHPB tests, an automatic single surface contact is employed for the contact between two elastic bars and rocklike specimen. Automatic single surface contact is the simplest type of contact with no definition of contact or target surface, and LS-DYNA automatically determines which surfaces within a model may come into contact. As lubricant, such as Vaseline, is applied on both ends of rocklike specimen in physical SHPB tests, the friction effect between elastic bars and rocklike specimen is eliminated. Therefore, the interfacial friction effect is negligible in numerical simulations.

ANSYS is used to prepare 3D finite element models, and the SOLID164 element with one integration point is employed to save the computer time $[15,21]$. The SOLID164 element is an eight-node solid hexahedron element in explicit dynamic analyses. After establishing 3D finite element model, a keyword file is output from ANSYS and is modified for LS-DYNA by applying the Holmquist-Johnson-Cook (HJC) model to the rocklike specimen. In numerical simulations, the mesh sensitivity is evaluated by a dimensionless mesh parameter, the ratio of smallest model size to largest element size. According to the research of Kariem et al. [12], numerical simulation results is insensitive to the mesh smaller than $1.5 \mathrm{~mm}$. Considering the smallest model size is the diameter, $12.7 \mathrm{~mm}$, the critical dimensionless mesh parameter is about 8.5 for SHPB numerical simulation. Then, a dimensionless mesh parameter of 10 is chosen and both incident bar and transmitted bar consist of 60000 hexahedron elements. Considering small nonparallel end face, a finer mesh is employed for the rocklike specimen and rocklike specimen consists of 60000 hexahedron elements. Hence, a total of 180000 hexahedron elements are involved in a 3D finite element model.

In physical SHPB tests, a compressive loading stress wave is generated by launching a striker impacting on the incident bar. For traditional rectangular compressive stress wave, premature failure of the rocklike specimen before stress equilibrium makes test results unreliable. Moreover, high signal oscillation presents in rectangular compressive stress wave due to the wave dispersion [22]. Therefore, the traditional rectangular compressive loading stress wave should be modified. Half-sine loading stress wave generated by a cone-shape striker proves to be a suitable, rational, and effective waveform for rocklike materials with good immunity to premature failure before stress equilibrium, geometric dispersion effect, and Pochhammer-Chree oscillation [11, 22-24]. Moreover, half-sine loading stress wave gives the possibility to an approximate constant strain rate condition. According to the typical example of dynamic stress balance analysis in ISRM suggested test methods [11], the amplitude and duration of half-sine loading stress for numerical simulation is assumed to be $260 \mathrm{MPa}$ and $240 \mu \mathrm{s}$, respectively. As no striker is in the $3 \mathrm{D}$ finite element model, the half-sine loading stress wave is straightly loaded on the front-end face of the incident bar. 


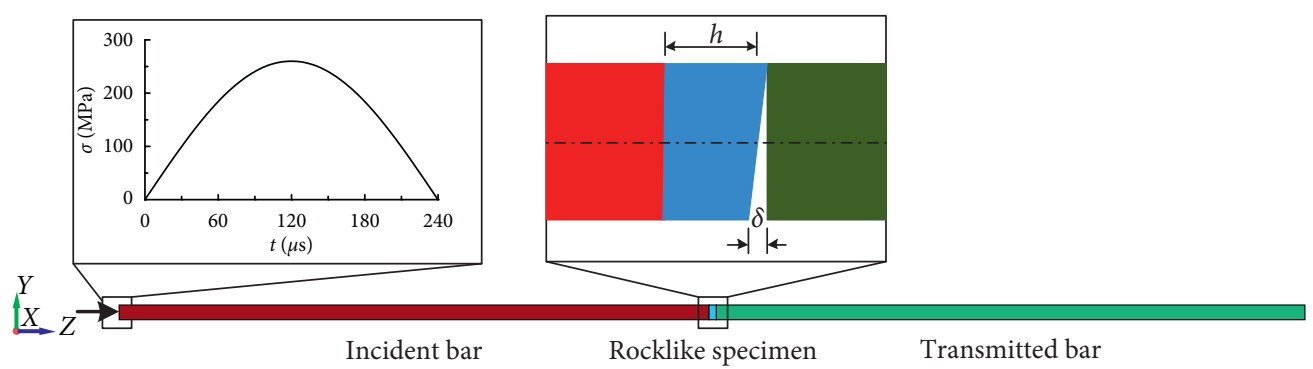

FIgURE 1: A schematic diagram of SHPB setup in the 3D finite element model.

The rocklike specimen with a length to diameter ratio of 0.5 is modeled and sandwiched between the incident bar and transmitted bar [11]. During specimen processing, the end faces of the rocklike specimen can be easily grinded to be smooth and flat. As the length to diameter ratio is just 0.5 , it is very difficult to control the parallelism between two end faces. End-face nonparallelism, $\gamma$, which is a measure of nonparallel deviation, is defined as the ratio of maximum height deviation $\delta$ to average height $h$, and it varies from $0 \%$ to $0.40 \%$ with an increment of $0.05 \%[15,21]$. Hence, the corresponding maximum height deviation ranges from $0 \mathrm{~mm}$ to $0.10 \mathrm{~mm}$.

2.2. Constitutive Models and Material Parameters. As for the dynamic characteristics test of rocklike materials, the incident and transmitted bars in physical SHPB apparatus are all made of a homogenous and isotropic alloy steel, and they keep in a linear elastic deformation state during SHPB tests. Therefore, the elastic constitutive model for an isotropic elastic material in LS-DYNA is selected for both incident and transmitted bars. According to the alloy steel properties in physical SHPB apparatus, the density, Young's modulus, and Poisson's ratio are set as $7.85 \mathrm{~g} / \mathrm{cm}^{3}, 210 \mathrm{GPa}$, and 0.30 , respectively.

Considering the high strain rate in SHPB tests, the HJC constitutive model, for materials subjected to large strain, high strain rate, and high pressure in LS-DYNA, is employed for the rocklike specimen [15, 25-27]. Young's modulus varies from $14 \mathrm{GPa}$ to $42 \mathrm{GPa}$ with an increment of $7 \mathrm{GPa}$. When Young's modulus of rocklike material is $28 \mathrm{GPa}$, material parameters of HJC constitutive model are shown in Table 1.

As five kinds of Young's moduli is considered, related material parameters of the HJC constitutive model should be modified with Young's modulus. In the HJC constitutive model, shear modulus $G$ and crushing volumetric strain $\mu_{\mathrm{C}}$ change with Young's modulus, and they can be expressed as follows:

$$
\begin{aligned}
G & =\frac{E}{2(1+v)}, \\
\mu_{C} & =\frac{p_{C}}{K}=\frac{p_{C}(1-2 v)}{E} .
\end{aligned}
$$

2.3. Verification of 3D Numerical Model. In line with the physical SHPB test, four hexahedron elements at the same cross section referring to pair strain gages symmetrically mounted on the surface of the bars are chosen to export $Z$ direction stress-time histories for stress uniformity analyses [21]. When Young's modulus is $28 \mathrm{GPa}$, acquired incident stress $\sigma(t)_{\mathrm{I}}$, reflected stress $\sigma(t)_{\mathrm{R}}$, and transmitted stress $\sigma(t)_{\mathrm{T}}$ for parallel rocklike specimen are illustrated in Figure 2.

As shown in Figure 2, the acquired incident stress wave is consistent with applied half-sine incident loading stress wave. Compared with the SHPB test results in ISRM suggested test methods [11] and literature [23], it can be found that the waveforms of acquired stress waves are similar with SHPB test results and a clear flat region is also presented in reflected stress wave. Slight difference is the result of different incident stress amplitudes and HJC material parameters. When failure occurs, deleting the failure rocklike specimen in numerical simulation leads to stress wave reflecting to incident bar, which causes a second peak in the reflected stress wave.

The fundamental assumptions of SHPB technique are one-dimensional stress wave propagation and stress uniformity $[1,7,10]$. To verify the numerical simulation results of SHPB tests, stress-time histories at two ends of rocklike specimens are compared and the unbalance stress is also checked [21, 28, 29]. Numerical model verifications are performed for parallel end-face rocklike specimen with Young's modulus of $14 \mathrm{GPa}, 21 \mathrm{GPa}, 28 \mathrm{GPa}, 35 \mathrm{GPa}$, and $42 \mathrm{GPa}$. According to acquired incident stress $\sigma(t)_{\mathrm{I}}$, reflected stress $\sigma(t)_{\mathrm{R}}$, and transmitted stress $\sigma(t)_{\mathrm{T}}$ exporting from numerical simulations, the stress-time histories on two ends of parallel end-face rocklike specimens are illustrated in Figure 3. In Figure 3, the unbalance stress, defined as $\sigma(t)_{\mathrm{I}}+\sigma(t)_{\mathrm{R}}-\sigma(t)_{\mathrm{T}}$, is also presented.

As shown in Figure 3, stress-time histories on two ends of parallel end-face rocklike specimens are basically the same, and the unbalanced stress is very small and can be omitted. Therefore, the stress uniformity state is achieved during numerical simulation, and the $3 \mathrm{D}$ finite element model is valid for the following energy dissipation analyses. Besides, the duration of transmitted stress wave extends with the increase of Young's modulus of the HJC constitutive model.

\section{Energy Dissipation Analyses during SHPB Tests}

3.1. Energy Evolution Analyses. When half-sine loading stress wave propagates in an elastic steel bar, both elastic deformation and motion are generated in elastic steel bars. 
TABLE 1: Material parameters of HJC constitutive model for rocklike material $[15,25]$.

\begin{tabular}{lccccccccc}
\hline$\rho\left(\mathrm{g} / \mathrm{cm}^{3}\right)$ & $F_{\mathrm{C}}(\mathrm{GPa})$ & $A$ & $B$ & $C$ & $\mathrm{SF}_{\mathrm{MAX}}$ & $G(\mathrm{GPa})$ & $D_{1}$ & $D_{2}$ & $N$ \\
\hline 2.47 & 0.13 & 0.79 & 1.60 & 0.007 & 4.0 & 11.67 & 0.045 & 1.0 & 0.61 \\
\hline $\mathrm{EF}_{\mathrm{MIN}}$ & $T(\mathrm{MPa})$ & $p_{\mathrm{C}}(\mathrm{MPa})$ & $\mu_{\mathrm{C}}$ & $p_{\mathrm{L}}$ & $\mu_{\mathrm{L}}$ & $K_{1}(\mathrm{GPa})$ & $K_{2}(\mathrm{GPa})$ & $K_{3}(\mathrm{GPa})$ & $F_{\mathrm{S}}$ \\
\hline 0.005 & 7.07 & 43.33 & 0.00278 & 1 & 0.1 & 85 & -171 & 208 & 0.004 \\
\hline
\end{tabular}

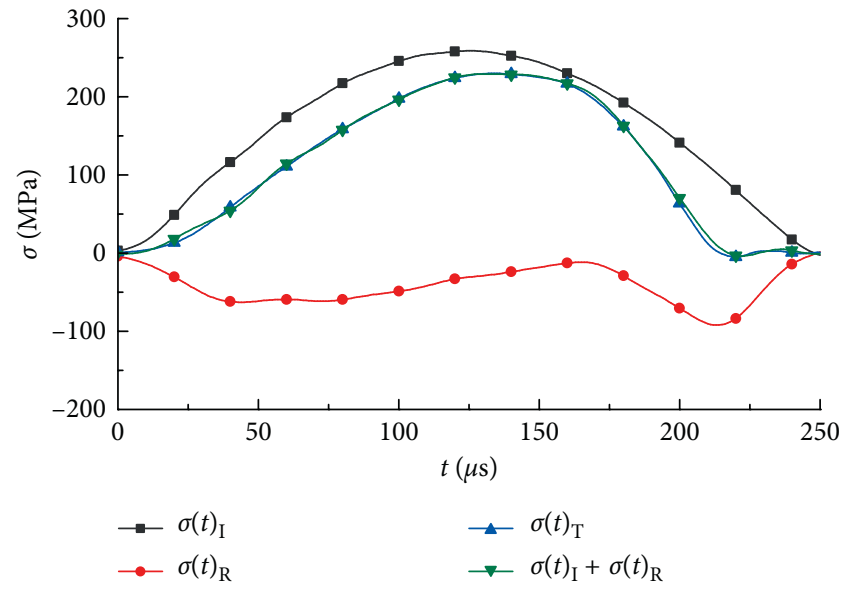

Figure 2: Acquired incident, reflected, and transmitted stresses when Young's modulus is $28 \mathrm{GPa}$.

The energy carried by stress wave is composed of elastic strain energy and kinetic energy, and the elastic strain energy is basically equal to the kinetic energy for the elastic stress wave [30]. The energy carried by incident stress wave, reflected stress wave, and transmitted stress wave can be calculated as follows $[18,19,30]$ :

$$
W_{i}=\frac{A C}{E} \int \sigma(t)_{i}^{2} d t, \quad i=I, R, T,
$$

where $\sigma(t)$ and $\varepsilon(t)$ denote the stress-time history and straintime history in elastic bars, and the subscript $i$ can be $I, R$, and $T$, which refer to incident stress wave, reflected stress wave, and transmitted stress wave, respectively. $E, A$, and $C$ are the Young's modulus, cross-sectional area, and longitudinal wave velocity of elastic bars, respectively.

An isothermal process is assumed in SHPB tests, and there is no heat exchange with the external environment. According to the first law of thermodynamics, also known as the energy conservation law, the absorption energy of rocklike specimen can be expressed as follows by neglecting the energy loss in SHPB tests [31, 32]:

$$
\begin{aligned}
W_{\mathrm{L}}= & W_{\mathrm{I}}-W_{\mathrm{R}}-W_{\mathrm{T}}=\frac{A C}{E} \int \sigma(t)_{\mathrm{I}}^{2} d t \\
& -\frac{A C}{E} \int \sigma(t)_{\mathrm{R}}^{2} d t-\frac{A C}{E} \int \sigma(t)_{\mathrm{T}}^{2} d t .
\end{aligned}
$$

According to the fundamental assumption of SHPB technique, stress uniformity, equation (3) can be rewritten as follows:

$$
W_{\mathrm{L}}=\frac{A C}{E} \int 2 \sigma(t)_{\mathrm{R}} \sigma(t)_{\mathrm{T}} \mathrm{d} t
$$

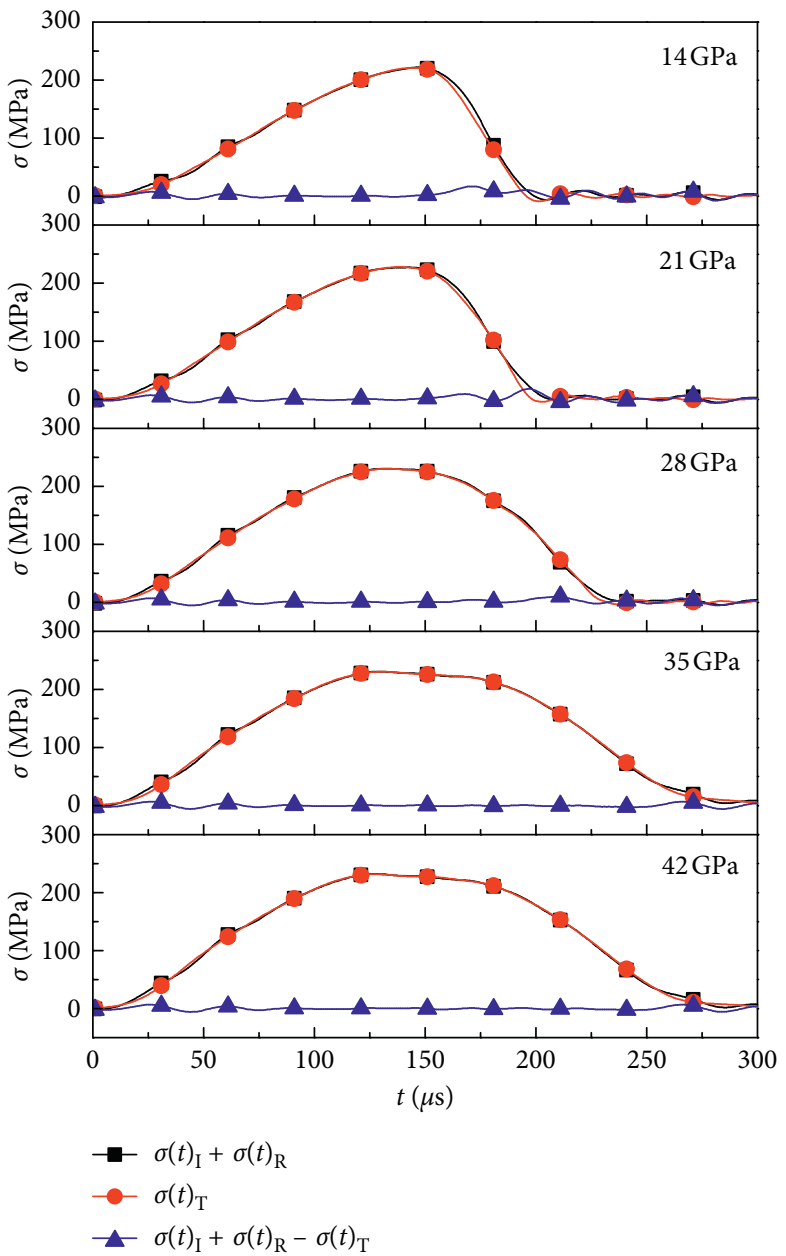

Figure 3: Stress-time histories on two ends of parallel end-face rocklike specimens.

Based on equations (2) and (4), time histories of incident energy, reflected energy, transmitted energy, and absorption energy for parallel end-face rocklike specimen with Young's modulus of $28 \mathrm{GPa}$ are illustrated in Figure 4.

As illustrated in Figure 4, the foregoing energies increase with the increment of loading time, and the transmitted energy accounts for most of incident energy.

3.2. Effect of Nonparallel End Face on Reflected, Transmitted, and Absorption Energies. As only one type of half-sine loading stress wave is considered, the incident energy, namely, the whole input energy, is a constant value, $388.78 \mathrm{~J}$. Based on SHPB numerical simulations, the variation of reflected energy and transmitted energy with the increment of end-face nonparallelism $\gamma$ is illustrated in Figure 5. 


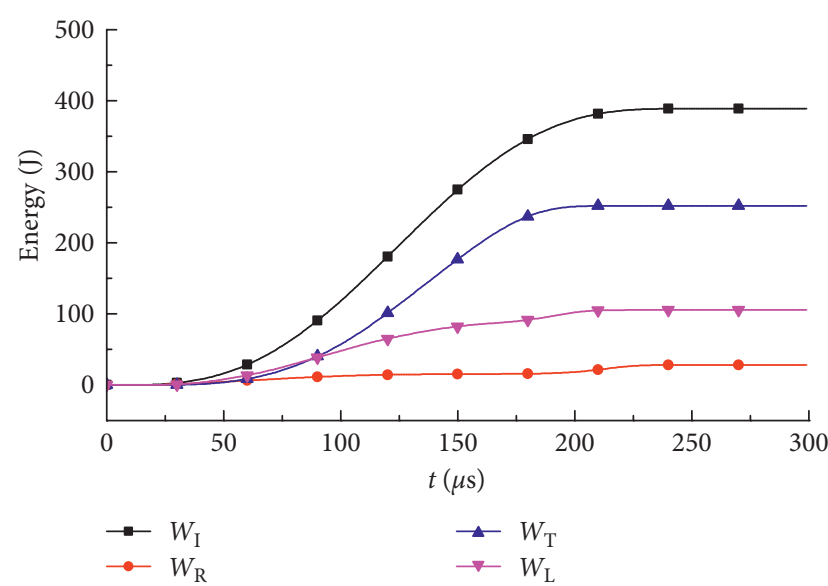

Figure 4: Energy-time histories during SHPB numerical simulation.

As shown in Figure 5, with the increment of end-face nonparallelism, reflected energy shows a slight increase trend, while transmitted energy presents a slight decrease trend. Moreover, for a constant end-face nonparallelism, the reflected energy decreases with the increase of Young's modulus, while the transmitted energy shows an inverse trend. These results are consistent with the characteristics of stress wave propagation. As described in the research of Yuan et al. [21], the amplitude of reflected stress wave gradually rises with the increment of end-face nonparallelism and decreases with the increase of Young's modulus, while the amplitude of transmitted stress wave shows an opposite variation trend.

Figure 6 shows the variation of total absorption energy with the increment of end-face nonparallelism.

As illustrated in Figure 6, total absorption energy shows a general upward trend with the increment of end-face nonparallelism and an overall downward trend with the increase of Young's modulus of the HJC constitutive model. When Young's modulus is within $28 \mathrm{GPa}$, there is almost a linear relation between total absorption energy and end-face nonparallelism, and the slope of the linear trend for Young's modulus of $14 \mathrm{GPa}, 21 \mathrm{GPa}$, and $28 \mathrm{GPa}$ is small and roughly the same. While for Young's modulus of $35 \mathrm{GPa}$ and $42 \mathrm{GPa}$, a rapid increase of total absorption energy is followed by a slow linear variation. According to the dynamic stress-strain curve [15], there is an elastic unloading and total strain shrinkage due to slight broken or damage of the rocklike specimen. As a result of the presence of elastic unloading, the absorption energy releases during the unloading phase. The rapid increase of total absorption energy indicates a quick decrease of released absorption energy. As energy dissipation is closely related with damage evolution, the total damage of the rocklike specimen after SHPB tests also increases rapidly with end-face nonparallelism increasing from $0.20 \%$ to $0.35 \%$ for Young's modulus of $35 \mathrm{GPa}$ and from $0.30 \%$ to $0.40 \%$ for Young's modulus of $42 \mathrm{GPa}$.

3.3. Characteristics of Energy Density Evolution. To illustrate the effect of energy dissipation per unit volume, energy

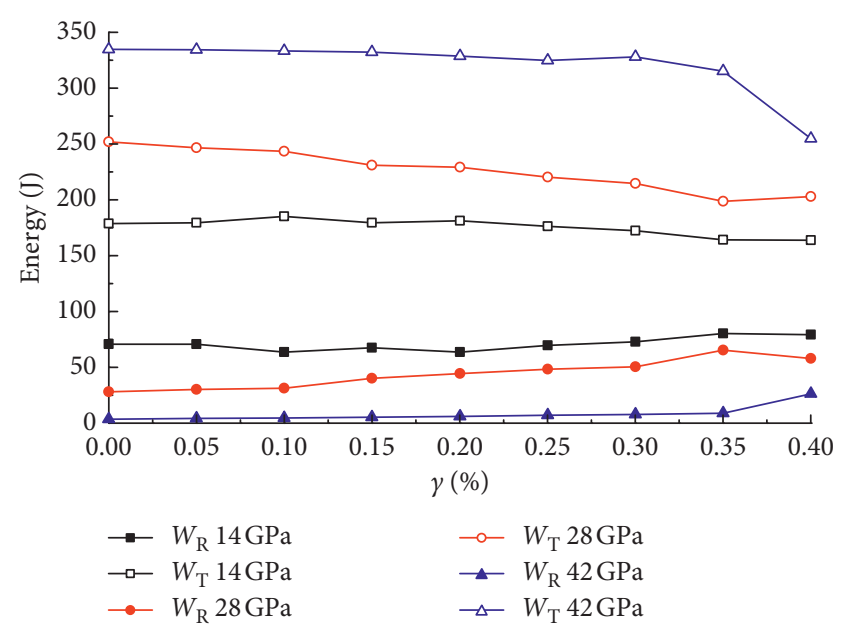

FIGURE 5: Variation of reflected energy and transmitted energy with end-face nonparallelism.

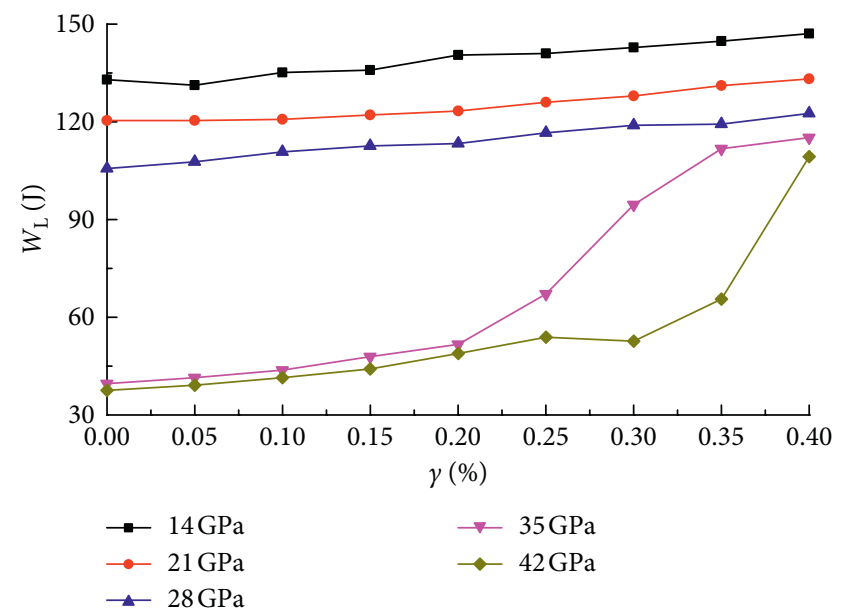

Figure 6: Variation of total absorption energy with end-face nonparallelism.

consumption density, also known as specific energy absorption, is defined as the energy consumed for breaking the rocklike specimen per unit volume. Therefore, the energy consumption density presents a similar variation trend to absorption energy. In one-dimensional loading condition, energy consumption density is defined as the area of dynamic stress-strain curve and can by calculated as follows:

$$
\begin{aligned}
U & =\frac{W_{\mathrm{L}}}{V_{\mathrm{s}}}=\int \sigma d \varepsilon=\int \sigma \dot{\varepsilon} d t=\int \sigma(t)_{\mathrm{T}} \frac{2 C}{l_{\mathrm{s}}} \frac{\sigma(t)_{\mathrm{R}}}{E} d t \\
& =\frac{C}{E l_{\mathrm{s}}} \int 2 \sigma(t)_{\mathrm{T}} \sigma(t)_{\mathrm{R}} d t,
\end{aligned}
$$

where $V_{\mathrm{s}}$ and $l_{\mathrm{s}}$ are the volume and length of the rocklike specimen and $U$ is the energy consumption density.

According to the research of Wang et al. [20], the energy consumption of a rocklike material consists of dissipated energy and releasable elastic strain energy. The releasable elastic strain energy density and dissipated energy density can be calculated as follows: 


$$
\begin{aligned}
U^{\mathrm{e}} & =\frac{1}{2} \sigma \varepsilon^{\mathrm{e}}=\frac{1}{2} \frac{\sigma(t)_{\mathrm{T}}^{2}}{E_{\mathrm{d}}}, \\
U^{\mathrm{d}} & =U-U^{\mathrm{e}},
\end{aligned}
$$

where $E_{\mathrm{d}}$ is dynamic Young's modulus of rocklike specimen, which can be obtained from the dynamic stress-strain curve and $U^{\mathrm{e}}$ and $U^{\mathrm{d}}$ are the releasable elastic strain energy density and dissipated energy density, respectively.

Based on equations (5)-(7), the energy density evolution curves of the rocklike specimens are illustrated in Figure 7.

Obviously from Figure 7, both Young's modulus of the HJC constitutive model and end-face nonparallelism affect energy density evolution. On the one hand, both energy consumption density and dissipated energy density increase with the increment of end-face nonparallelism, while releasable elastic strain energy density reduces slightly. On the other hand, all three energy densities decrease with the increase of Young's modulus of the HJC constitutive model. It is worth mentioning that the presence of elastic unloading in slight broken or damaged rocklike specimen leads to an obvious total strain shrinkage after peak dynamic stress, which causes release of elastic strain energy density and reduction of energy consumption density. Therefore, the dissipated energy density is approximately a constant value with the shrinkage of total strain, and the final dissipated energy density increases with the increment of end-face nonparallelism.

Due to the nonparallel end face, fluctuation presents in reflected stresses and transmitted stresses [21]. Hence, fluctuation also presents in the evolution of both releasable elastic strain energy density and dissipated energy density. The larger the end-face nonparallelism is, the greater the fluctuation appears. The larger the Young's modulus is, the weaker the fluctuation is.

3.4. Energy Dissipation and Elastic Energy Release at Peak Dynamic Stress. Under uniaxial compression, a typical complete stress-strain curve consists of five stages, crack closure, elastic, cracking, postfailure, and residual, and the idealized stress-strain curve can be basically divided into two regions, prefailure region and postfailure region [33]. In numerical simulation, both the crack closure and residual stages cannot be presented for no crack in the finite element model and elements deleted after failure. Therefore, at the end of postfailure, the dissipated energy density is basically equal to the energy consumption density due to residual stress approaching zero. The critical point between elastic and cracking stages is difficult to determine, while the critical point between cracking or prefailure and postfailure is easy to determine, which is the peak dynamic stress. Considering the critical point between prefailure and postfailure regions, the detail energy densities at the peak dynamic stress are calculated and listed in Table 2. The releasable elastic strain energy density and dissipated energy density at the peak dynamic stress are marked as $U_{\mathrm{p}}^{\mathrm{e}}$ and $U_{\mathrm{p}}^{\mathrm{d}}$, respectively.

As shown in Table 2, dissipated energy density at the peak dynamic stress presents a linear upward trend with the increment of end-face nonparallelism and Young's modulus, while releasable elastic strain energy density at the peak dynamic stress shows a linear downward trend. The smaller the Young's modulus is, the more obvious the effect of endface nonparallelism is.

In order to study the influence of nonparallel end face on energy dissipation characteristics, the energy dissipation ratio $K_{\mathrm{p}}$ is defined as dividing dissipated energy density by energy consumption density at the peak dynamic stress and can be calculated as follows:

$$
K_{\mathrm{p}}=\frac{U_{\mathrm{p}}^{\mathrm{d}}}{U_{\mathrm{p}}}
$$

With the increment of end-face nonparallelism, energy dissipation ratios for various Young's moduli are drawn in Figure 8 .

As clearly illustrated in Figure 8, the energy dissipation ratio increases linearly with the increment of end-face nonparallelism, and the growth trends for various Young's moduli are approximate parallel to each other. The smaller Young's modulus is, the less the energy dissipation ratio is. Linear regression equations with constant slope and different intercepts are employed. Linear regression equations can be expressed as follows:

$$
K_{\mathrm{p}}=0.23 \gamma+C
$$

where $C$ is the intercept of linear regression equation.

Intercept $C$ is closely related with Young's modulus of the HJC constitutive model. There is also a linear relation between the intercept $C$ and Young's modulus. A linear regression equation is also employed by a dimensionless Young's modulus $E^{\prime}$ and can be expressed as follows:

$$
C=0.22 E^{\prime}+0.129=0.22 \frac{E-E_{\min }}{E_{\max }-E_{\min }}+0.129 \text {, }
$$

where $E_{\max }$ and $E_{\min }$ are maximum and minimum value of considered Young's modulus, which are $42 \mathrm{GPa}$ and $14 \mathrm{GPa}$, respectively.

Hence, with end-face nonparallelism and dimensionless Young's modulus as variables, a binary linear regression equation is deduced for energy dissipation ratio and is expressed as follows:

$$
K_{\mathrm{p}}=0.23 \gamma+0.22 E^{\prime}+0.129
$$

\section{Mechanical Damage Evolution Based on Energy Density Analyses}

As deformation and failure progress of the rocklike material is also the progress of energy dissipation, mechanical damage can be defined as the ratio of dissipated energy density to total energy consumption density, which can be calculated as follows [20]:

$$
D=\frac{U^{d}}{U}
$$



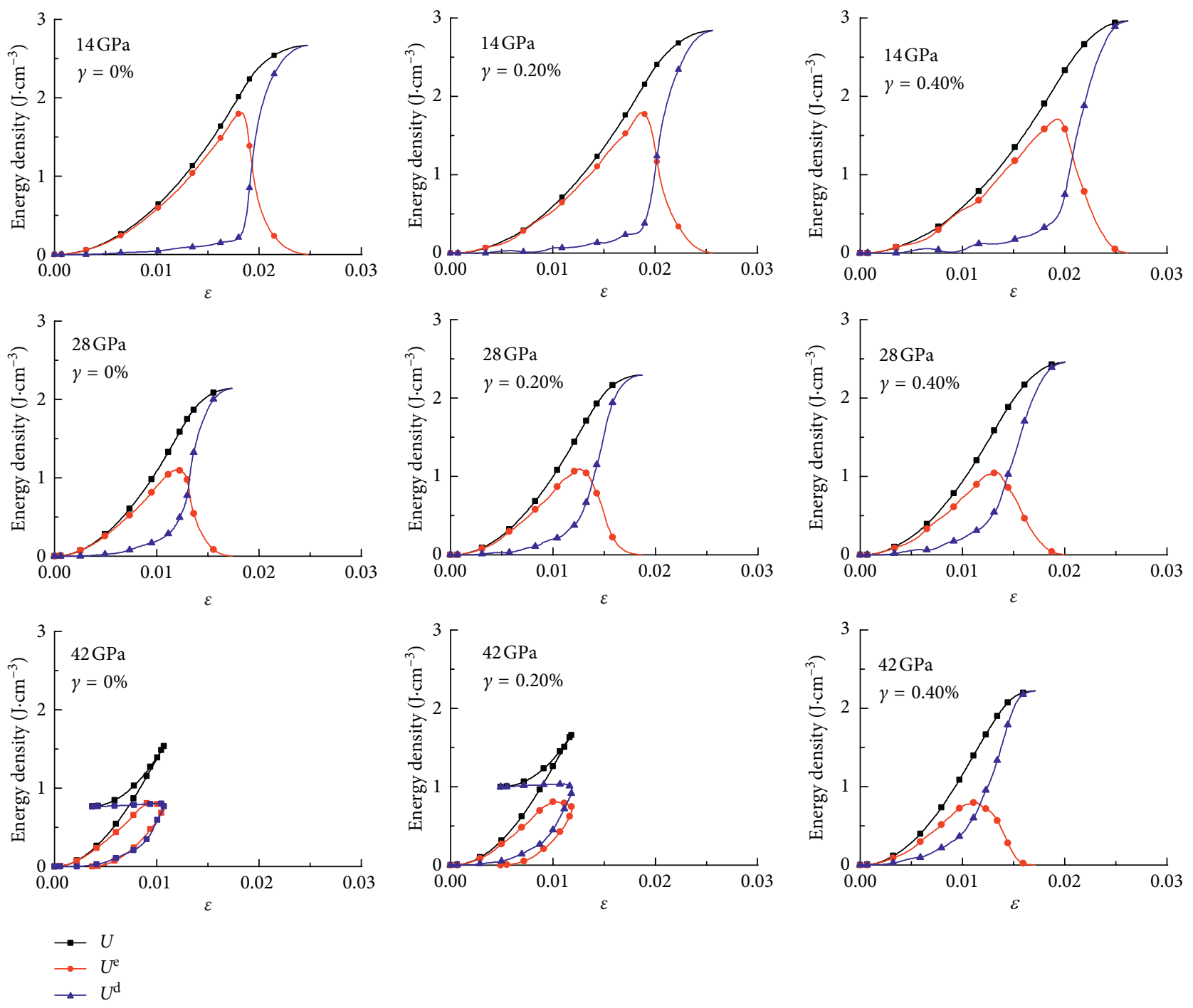

FiguRE 7: Energy density evolution curves of the rocklike specimen.

TABle 2: Releasable elastic strain energy density and dissipated energy density at peak dynamic stress (unit: $J \cdot \mathrm{cm}^{-3}$ ).

\begin{tabular}{lcccccrrrrr}
\hline$\gamma(\%)$ & \multicolumn{2}{c}{$14 \mathrm{GPa}$} & \multicolumn{2}{c}{$21 \mathrm{GPa}$} & \multicolumn{2}{c}{$28 \mathrm{GPa}$} & \multicolumn{2}{c}{$35 \mathrm{GPa}$} & \multicolumn{2}{c}{$42 \mathrm{GPa}$} \\
& $U_{\mathrm{p}}^{\mathrm{e}}$ & $U_{\mathrm{p}}^{\mathrm{d}}$ & $U_{\mathrm{p}}^{\mathrm{e}}$ & $U_{\mathrm{p}}^{\mathrm{d}}$ & $U_{\mathrm{p}}^{\mathrm{e}}$ & $U_{\mathrm{p}}^{\mathrm{d}}$ & $U_{\mathrm{p}}^{\mathrm{e}}$ & $U_{\mathrm{p}}^{\mathrm{d}}$ & $U_{\mathrm{p}}^{\mathrm{e}}$ & $U_{\mathrm{p}}^{\mathrm{d}}$ \\
\hline 0 & 1.808 & 0.260 & 1.372 & 0.344 & 1.101 & 0.416 & 0.947 & 0.409 & 0.818 \\
0.05 & 1.802 & 0.254 & 1.368 & 0.378 & 1.100 & 0.431 & 0.941 & 0.453 & 0.809 & 0.422 \\
0.10 & 1.803 & 0.319 & 1.356 & 0.394 & 1.098 & 0.420 & 0.937 & 0.445 & 0.804 & 0.459 \\
0.15 & 1.791 & 0.280 & 1.350 & 0.393 & 1.092 & 0.447 & 0.937 & 0.450 & 0.806 & 0.462 \\
0.20 & 1.790 & 0.303 & 1.343 & 0.407 & 1.092 & 0.468 & 0.937 & 0.471 & 0.809 \\
0.25 & 1.786 & 0.348 & 1.342 & 0.415 & 1.093 & 0.473 & 0.936 & 0.492 & 0.808 & 0.476 \\
0.30 & 1.773 & 0.384 & 1.330 & 0.448 & 1.081 & 0.507 & 0.938 & 0.526 & 0.805 \\
0.35 & 1.742 & 0.439 & 1.316 & 0.483 & 1.070 & 0.539 & 0.935 & 0.565 & 0.808 \\
0.40 & 1.708 & 0.471 & 1.286 & 0.534 & 1.051 & 0.582 & 0.923 & 0.599 & 0.548 \\
\hline
\end{tabular}

For a certain dynamic stress-strain curve, the total energy consumption density $U$ is a definite value. For rocklike specimens with total strain shrinkage in the dynamic stressstrain curve, total energy consumption density is reduced during the release of elastic strain energy in total strain shrinkage. If total energy consumption density is used for the rocklike specimen with strain shrinkage, the mechanical damage is approximate to 1 , which is inconsistent with the slight broken or damaged rocklike specimen in numerical simulations. Hence, energy consumption density at the peak 


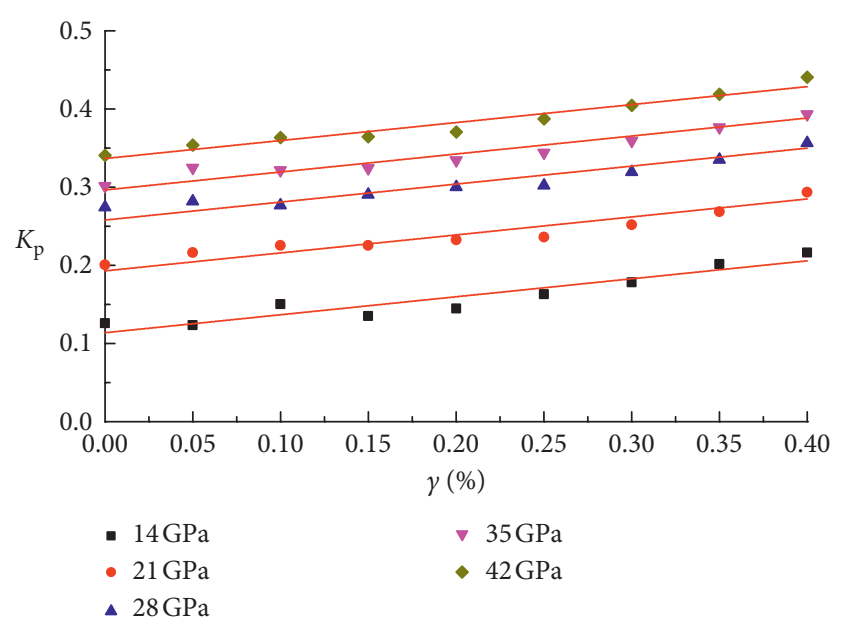

FIGURE 8: Energy dissipation ratio versus end-face nonparallelism.

value of the evolution curve is employed to calculate the mechanical damage. The energy consumption densities for mechanical damage calculation are listed in Table 3.

Figure 9 shows the mechanical damage evolution for various end-face nonparallelism and Young's moduli. The shape of mechanical damage evolution curve is similar to that of dissipated energy density.

As clearly seen from Figure 9, the mechanical damage evolution is influenced by both end-face nonparallelism and Young's modulus of the HJC constitutive model. In line with two regions in the dynamic stress-strain curve, prefailure, and postfailure, the mechanical damage evolution of the rocklike specimen in the dynamic loading condition can also be divided into to two regions, slow-growth region and rapid-growth region, especially in small Young's modulus. In slow-growth region, fluctuation presents in mechanical damage evolution due to the existence of nonparallel end face. The smaller the Young's modulus is, the more remarkable the fluctuation is. In the rapid-growth region, the end-face nonparallelism shows little influence on mechanical damage development, while Young's modulus demonstrates an impact on mechanical damage development. The smaller the Young's modulus is, the more rapidly the mechanical damage develops.

Transition between two regions of damage evolution is obvious and easy to determine in small Young's modulus, while it becomes difficult to determine in large Young's modulus, as illustrated in Figure 9. With the increment of end-face nonparallelism, the transition is shifted to the right, which indicates an increase of both strain and damage threshold value at the transition. While with the increase of Young's modulus, the strain at the transition decreases and the damage threshold value at the transition increases and this may be the result of decreasing crushing volumetric strain $\mu_{\mathrm{C}}$ with Young's modulus of the HJC constitutive model.

\section{Discussion on Allowable Processing Deviation}

For parallel end face rocklike specimens, the dynamic stressstrain curve for various Young's moduli is illustrated in Figure 10.
TABle 3: Energy consumption density for mechanical damage calculation (unit: $\mathrm{J} \cdot \mathrm{cm}^{-3}$ ).

\begin{tabular}{lccccc}
\hline$\gamma(\%)$ & & \multicolumn{5}{c}{} \\
\hline 0 & 2.658 & 2.439 & 2.142 & 1.630 & 1.546 \\
0.05 & 2.627 & 2.427 & 2.190 & 1.661 & 1.576 \\
0.10 & 2.728 & 2.436 & 2.246 & 1.691 & 1.607 \\
0.15 & 2.731 & 2.462 & 2.273 & 1.725 & 1.641 \\
0.20 & 2.838 & 2.482 & 2.296 & 1.769 & 1.673 \\
0.25 & 2.846 & 2.526 & 2.337 & 1.904 & 1.729 \\
0.30 & 2.886 & 2.576 & 2.407 & 2.012 & 1.754 \\
0.35 & 2.930 & 2.646 & 2.407 & 2.272 & 1.895 \\
0.40 & 2.969 & 2.689 & 2.456 & 2.345 & 2.229 \\
\hline
\end{tabular}

As obvious in Figure 10, Young's modulus has a great impact on the shape of the dynamic stress-strain curve. When Young's modulus is $35 \mathrm{GPa}$ or $42 \mathrm{GPa}$, an elastic unloading with total strain shrinkage is presented, which indicates a slight breakage of the rocklike specimen. In the elastic unloading phase, the energy consumption decreases with total strain shrinkage due to the release of elastic strain energy. With the increment of end-face nonparallelism, the elastic unloading phase diminishes gradually; therefore, releasable elastic strain energy in elastic unloading also decreases. Hence, the reduction of energy consumption density for Young's modulus of $42 \mathrm{GPa}$ in Figure 7 decreases with the increment of end-face nonparallelism. With the continuous increases of end-face nonparallelism for Young's modulus of $35 \mathrm{GPa}$ and $42 \mathrm{GPa}$, the curve shape of both dynamic stress-strain and energy evolution is changed, and then the mechanical damage evolution is also changed, which makes the SHPB test results unreliable.

Dynamic characteristics, energy density evolution, and mechanical damage evolution are desired by conducting SHPB tests. Both nonparallel end face and Young's modulus have a great impact on SHPB test results of rocklike specimens. Young's modulus of the rocklike material is an intrinsic characteristic of pending tested rocklike materials, and it is unknown before the test. In order to make the SHPB test results reliable, the errors induced by the rocklike specimen processing deviation should be controlled within an acceptable level. It is infeasible to give an allowable processing deviation for various Young's moduli of rocklike materials. Therefore, a common practice is given an allowable processing deviation without regard to Young's modulus.

When end-face nonparallelism is $0.20 \%$, the curve shape of both energy density evolution and mechanical damage evolution remain unchanged, and the error induced by nonparallel end face is small. According to above analyses, maximum end-face nonparallelism can be controlled within $0.20 \%$, namely, the allowable processing deviation is $0.05 \mathrm{~mm}$ for $25 \mathrm{~mm}$ height rocklike specimen, which is twice the value in ISRM suggested methods [11]. Hence, the cost and time for processing rocklike specimens can be reduced.

\section{Conclusions}

Regarding nonparallel end face of rocklike specimens in SHPB tests, numerical simulations have been performed 

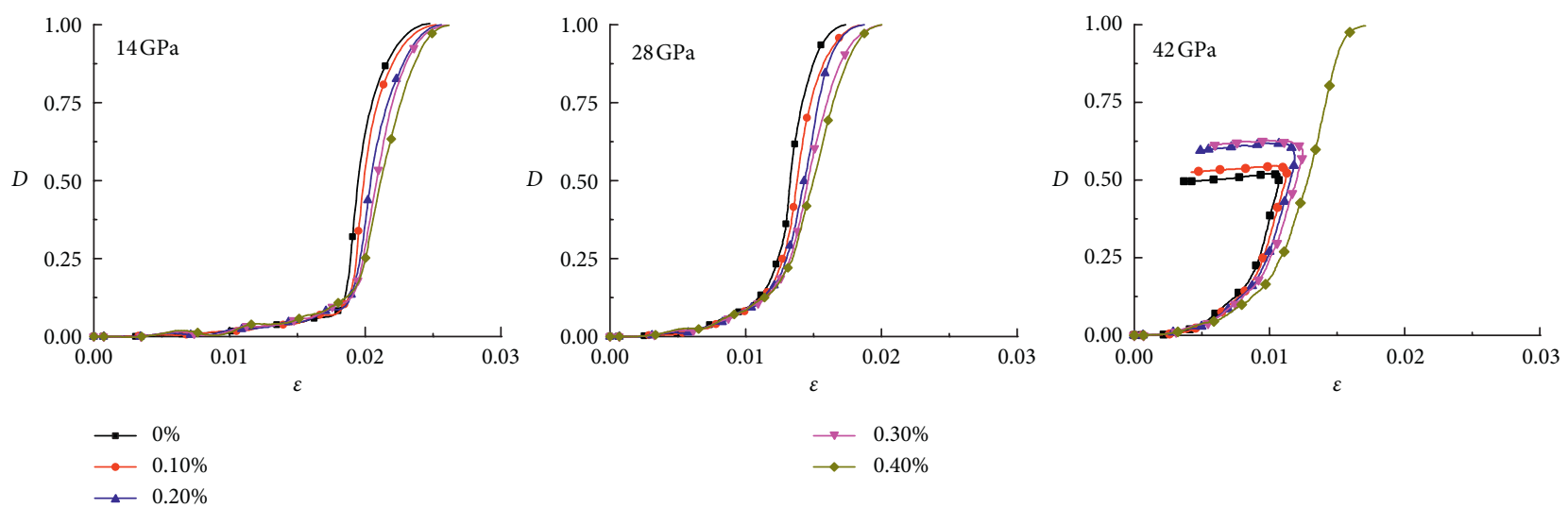

Figure 9: Mechanical damage evolution curves of the rocklike specimen.

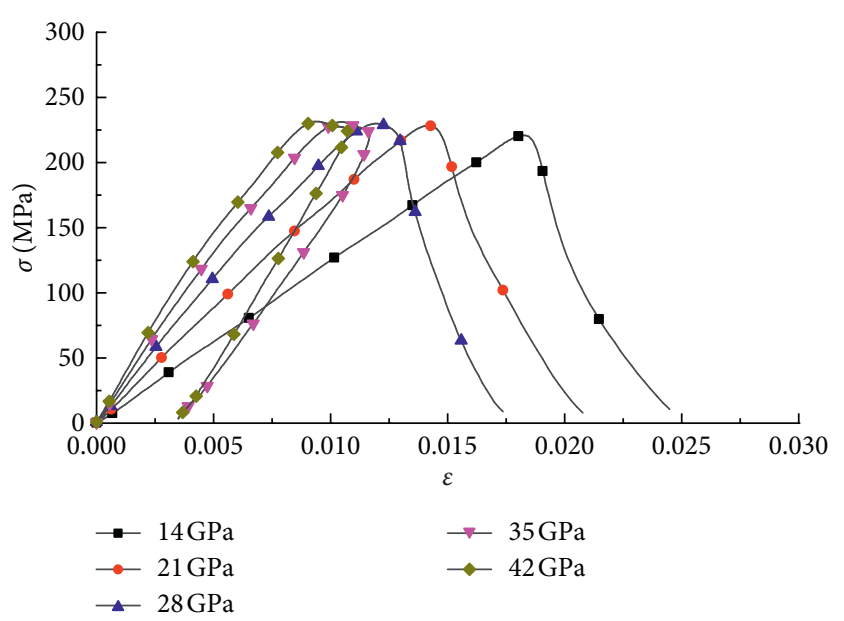

Figure 10: Dynamic stress-strain curve for various Young's moduli.

with end-face nonparallelism varying from $0 \%$ to $0.40 \%$ and Young's modulus ranging from $14 \mathrm{GPa}$ to $42 \mathrm{GPa}$. Then, the characteristics of energy dissipation and mechanical damage are analyzed to evaluate the effects of nonparallel end face. The main conclusions are summarized as follows:

(1) With the increment of end-face nonparallelism, both absorption energy and reflected energy show a slight increase trend, while transmitted energy presents a slight decrease trend.

(2) Both energy consumption density and dissipated energy density increase with the increment of endface nonparallelism, while releasable elastic strain energy density reduces slightly. Due to the presence of nonparallel end face, fluctuation presents in the evolution of both releasable elastic strain energy density and dissipated energy density. The fluctuation is enhanced with the increment of end-face nonparallelism and weakened with the increase of Young's modulus.

(3) At the peak dynamic stress, dissipated energy density presents a linear upward trend with the increment of end-face nonparallelism and Young's modulus, while releasable elastic strain energy density shows a linear downward trend. A binary linear regression equation is deduced to estimate energy dissipation ratio with end-face nonparallelism and Young's modulus.

(4) In line with two regions in the dynamic stress-strain curve, mechanical damage evolution of the rocklike specimen is also divided into to two regions, slowgrowth region and rapid-growth region. In the slow-growth region, fluctuation presents due to the presence of nonparallel end face, and it weakens with the increase of Young's modulus. Transition between two regions is shifted to the right with the increment of end-face nonparallelism, which indicates an increase of both strain and damage threshold values.

(5) Based on energy density evolution and mechanical damage evolution analyses, maximum end-face nonparallelism can be controlled within $0.20 \%$, namely, the allowable processing deviation is $0.05 \mathrm{~mm}$ for $25 \mathrm{~mm}$ height rocklike specimen. The suggested allowable processing deviation is twice the value in ISRM suggested methods, which reduces the cost and time for processing rocklike specimens.

\section{Data Availability}

The datasets generated and analyzed during the current study are available from the corresponding author on reasonable request.

\section{Conflicts of Interest}

The authors declare that there are no conflicts of interest regarding the publication of this paper.

\section{Acknowledgments}

This research was funded by the National Natural Science Foundation of China (no. 51774011), Anhui Provincial Natural Science Foundation (no. 1808085QE148), project funded by China Postdoctoral Science Foundation (no. 2018M642504), Natural Science Research Project of Colleges 
and Universities in Anhui Province (no. KJ2017A097), Young Teacher Scientific Research Project of Anhui University of Science and Technology (no. QN201607), Doctoral Fund Project of Anhui University of Science and Technology (no. 11674), Science and Technology Project of Department of Housing and Urban-Rural Development of Anhui Province (no. 2017YF-08), National Innovation and Entrepreneurship Training Program for College Students (no. 201810361029), and Anhui Provincial Innovation and Entrepreneurship Training Program for College Students (no. 201810361174).

\section{References}

[1] H. Kolsky, "An investigation of the mechanical properties of materials at very high rates of loading," Proceedings of the Physical Society: Section B, vol. 62, no. 11, pp. 676-700, 1949.

[2] S. Yadav, D. R. Chichili, and K. T. Ramesh, "The mechanical response of a 6061-T6 $\mathrm{A} 1 / \mathrm{Al}_{2} \mathrm{O}_{3}$ metal matrix composite at high rates of deformation," Acta Metallurgica et Materialia, vol. 43, no. 12, pp. 4453-4464, 1995.

[3] R. L. Woodward and R. H. Brown, "Dynamic stress-strain properties of a steel and a brass at strain rates up to 104 per second," Proceedings of the Institution of Mechanical Engineers, vol. 189, no. 1, pp. 107-115, 1975.

[4] C. A. Ross, P. Y. Thompson, and J. W. Tedesco, "SplitHopkinson pressure-bar tests on concrete and mortar in tension and compression," ACI Materials Journal, vol. 86, no. 5, pp. 475-481, 1989.

[5] G. Ravichandran and G. Subhash, "Critical appraisal of limiting strain rates for compression testing of ceramics in a split Hopkinson pressure bar," Journal of the American Ceramic Society, vol. 77, no. 1, pp. 263-267, 1994.

[6] D. Ma, Q. Ma, and P. Yuan, "SHPB tests and dynamic constitutive model of artificial frozen sandy clay under confining pressure and temperature state," Cold Regions Science and Technology, vol. 136, pp. 37-43, 2017.

[7] K. Xia and W. Yao, "Dynamic rock tests using split Hopkinson (Kolsky) bar system-a review," Journal of Rock Mechanics and Geotechnical Engineering, vol. 7, no. 1, pp. 27-59, 2015.

[8] B. Xie, D. Ai, and Y. Yang, "Crack detection and evolution law for rock mass under SHPB impact tests," Shock and Vibration, vol. 2019, Article ID 3956749, 12 pages, 2019.

[9] P. Baranowski, J. Malachowski, R. Gieleta, K. Damaziak, L. Mazurkiewicz, and D. Kolodziejczyk, "Numerical study for determination of pulse shaping design variables in SHPB apparatus," Bulletin of the Polish Academy of Sciences: Technical Sciences, vol. 61, no. 2, pp. 459-466, 2013.

[10] F. Dai, S. Huang, K. Xia, and Z. Tan, "Some fundamental issues in dynamic compression and tension tests of rocks using split Hopkinson pressure bar," Rock Mechanics and Rock Engineering, vol. 43, no. 6, pp. 657-666, 2010.

[11] Y. X. Zhou, K. Xia, X. B. Li et al., "Suggested methods for determining the dynamic strength parameters and mode-I fracture toughness of rock materials," International Journal of Rock Mechanics and Mining Sciences, vol. 49, pp. 105-112, 2012.

[12] M. A. Kariem, J. H. Beynon, and D. Ruan, "Misalignment effect in the split Hopkinson pressure bar technique," International Journal of Impact Engineering, vol. 47, pp. 60-70, 2012.
[13] X. Wu, Q. Yin, Y. Wei, and C. Huang, "Effects of imperfect experimental conditions on stress waves in SHPB experiments," Acta Mechanica Sinica, vol. 31, no. 6, pp. 827-836, 2015.

[14] R. Panowicz, J. Janiszewski, and K. Kochanowski, "Effects of sample geometry imperfections on the results of split Hopkinson pressure bar experiments," Experimental Techniques, pp. 1-7, 2018.

[15] P. Yuan and Q. Ma, "Correction of non-parallel end-faces of rock specimens in SHPB tests," Explosion and Shock Waves, vol. 37, no. 5, pp. 929-936, 2017.

[16] P. Yuan and Q. Y. Ma, "Split Hopkinson pressure bar tests on sandstone in coalmine under cyclic wetting and drying," Rock and Soil Mechanics, vol. 34, no. 9, pp. 2557-2562, 2013.

[17] H. P. Xie, R. D. Peng, Y. Ju, and H. W. Zhou, "Energy analysis of rock failure," Chinese Journal of Rock Mechanics and Engineering, vol. 24, no. 15, pp. 2603-2608, 2005.

[18] B. Lundberg, "A split Hopkinson bar study of energy absorption in dynamic rock fragmentation," International Journal of Rock Mechanics and Mining Sciences \& Geomechanics Abstracts, vol. 13, no. 6, pp. 187-197, 1976.

[19] J. Feng, E. Wang, R. Shen, L. Chen, X. Li, and Z. Xu, "Investigation on energy dissipation and its mechanism of coal under dynamic loads," Geomechanics and Engineering, vol. 11, no. 5, pp. 657-670, 2016.

[20] P. Wang, J. Xu, X. Fang, and P. Wang, "Energy dissipation and damage evolution analyses for the dynamic compression failure process of red-sandstone after freeze-thaw cycles," Engineering Geology, vol. 221, pp. 104-113, 2017.

[21] P. Yuan, Q. Y. Ma, and D. D. Ma, "Stress uniformity analyses on nonparallel end-surface rock specimen during loading process in SHPB tests," Advances in Civil Engineering, vol. 2018, Article ID 5406931, 12 pages, 2018.

[22] Z. Zhou, X. Li, A. Liu, and Y. Zou, "Stress uniformity of split Hopkinson pressure bar under half-sine wave loads," International Journal of Rock Mechanics and Mining Sciences, vol. 48, no. 4, pp. 697-701, 2011.

[23] Z. Y. Liao, J. B. Zhu, K. W. Xia, and C. A. Tang, "Determination of dynamic compressive and tensile behavior of rocks from numerical tests of split Hopkinson pressure and tension bars," Rock Mechanics and Rock Engineering, vol. 49, no. 10, pp. 3917-3934, 2016.

[24] G. P. Zou, X. H. Shen, Z. L. Chang, Y. W. Wang, and P. Wang, "A method of restraining the geometric dispersion effect on split-Hopkinson pressure bar by the modified striker bar," Experimental Techniques, vol. 40, no. 4, pp. 1249-1261, 2016.

[25] G. M. Zhao, W. W. Ma, and X. R. Meng, "Damage modes and energy characteristics of rock-like materials under dynamic load," Rock and Soil Mechanics, vol. 36, no. 12, pp. 3598-3605, 2015.

[26] G.-M. Ren, H. Wu, Q. Fang, and X.-Z. Kong, "Parameters of Holmquist-Johnson-Cook model for high-strength concretelike materials under projectile impact," International Journal of Protective Structures, vol. 8, no. 3, pp. 352-367, 2017.

[27] T. J. Holmquist, G. R. Johnson, and W. H. Cook, "A computational constitutive model for concrete subjected to large strains, high strain rates and high pressures," in Proceedings of the 14th International Symposium on Ballistics, Quebec City, Canada, September 1993.

[28] H. Zhao, "Material behaviour characterisation using SHPB techniques, tests and simulations," Computers \& Structures, vol. 81, no. 12, pp. 1301-1310, 2003.

[29] D. J. Frew, M. J. Forrestal, and W. Chen, "A split Hopkinson pressure bar technique to determine compressive stress-strain 
data for rock materials," Experimental Mechanics, vol. 41, no. 1, pp. 40-46, 2001.

[30] B. Song and W. Chen, "Energy for specimen deformation in a split Hopkinson pressure bar experiment," Experimental Mechanics, vol. 46, no. 3, pp. 407-410, 2006.

[31] Y. Deng, M. Chen, Y. Jin, and D. Zou, "Theoretical analysis and experimental research on the energy dissipation of rock crushing based on fractal theory," Journal of Natural Gas Science and Engineering, vol. 33, pp. 231-239, 2016.

[32] Y. Ju, H. Wang, Y. Yang, Q. Hu, and R. Peng, "Numerical simulation of mechanisms of deformation, failure and energy dissipation in porous rock media subjected to wave stresses," Science China Technological Sciences, vol. 53, no. 4, pp. 1098-1113, 2010.

[33] L. Tutluoğlu, İ. F. Öge, and C. Karpuz, "Relationship between pre-failure and post-failure mechanical properties of rock material of different origin," Rock Mechanics and Rock Engineering, vol. 48, no. 1, pp. 121-141, 2015. 


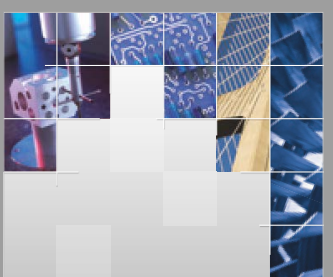

\section{Enfincering}
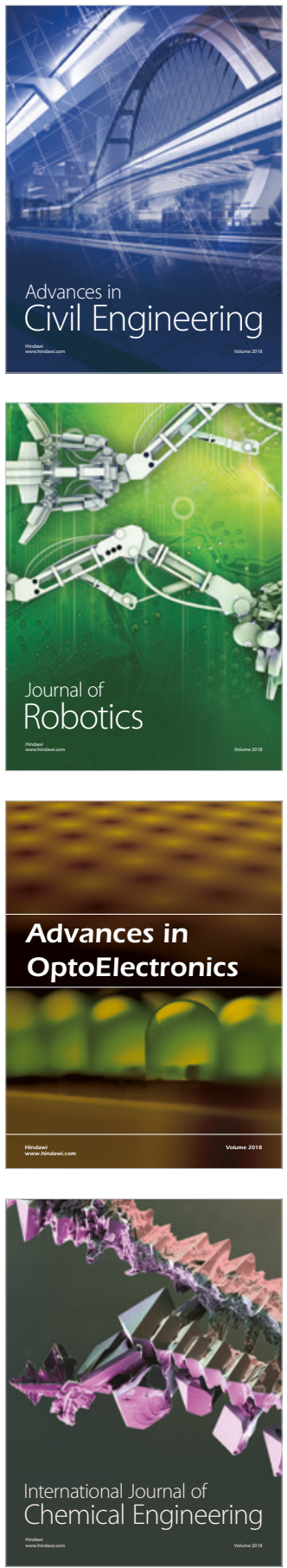

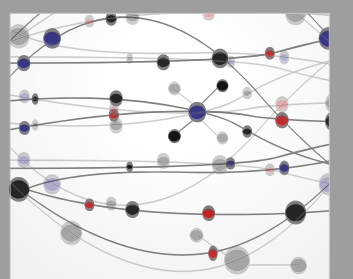

\section{Rotating \\ Machinery}

The Scientific World Journal

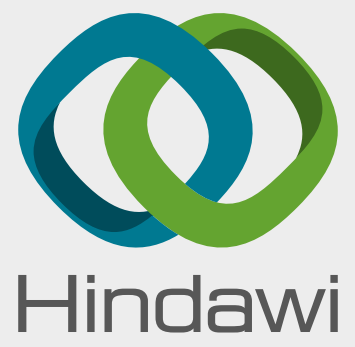

Submit your manuscripts at

www.hindawi.com
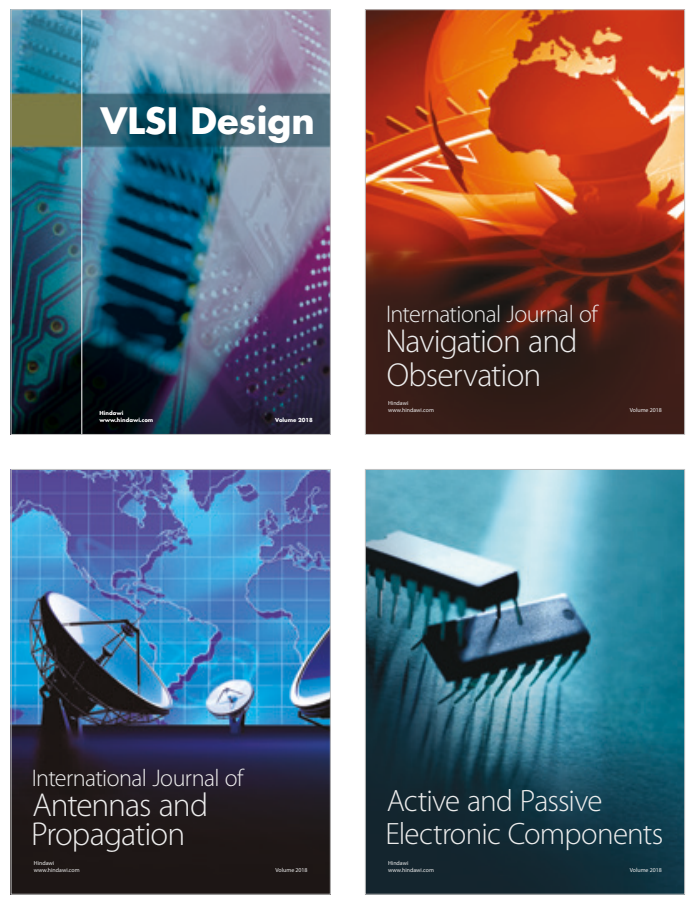
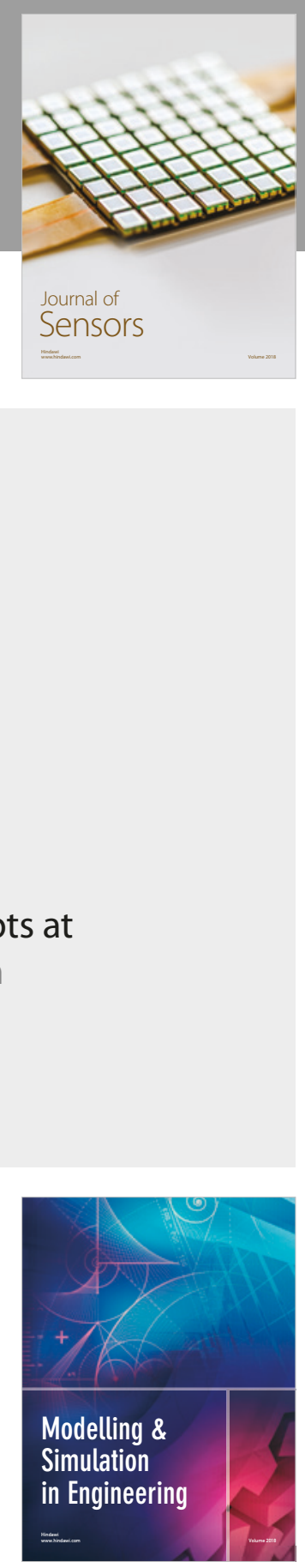

\section{Advances \\ Multimedia}
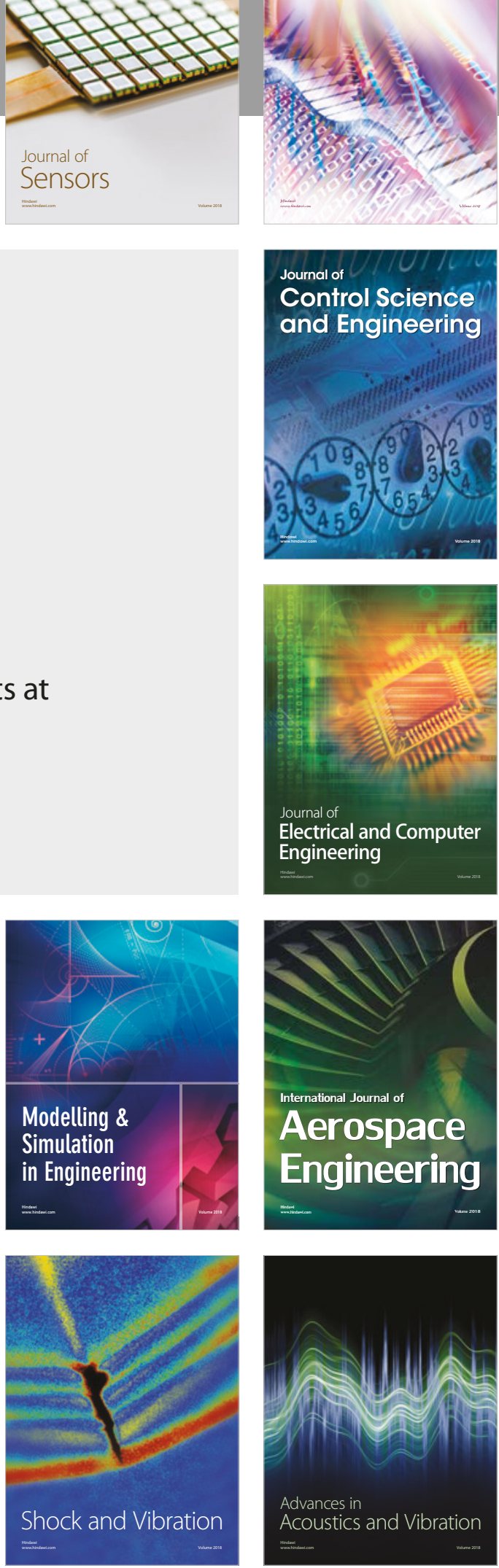